Artículo

\title{
Nanopartículas de quitosano mejoran la calidad nutracéutica de germinados de triticale
}

\author{
Silvia C. Ramírez Rodríguez ${ }^{1 \S}$ \\ Hortensia Ortega Ortiz ${ }^{2}$ \\ Manuel Fortis Hernández ${ }^{1}$ \\ Juan M. Nava Santos ${ }^{3}$ \\ Jorge A. Orozco Vidal ${ }^{1}$ \\ Pablo Preciado Rangel ${ }^{1}$ \\ ${ }^{1}$ Doctorado en Agua y Suelo-Instituto Tecnológico de Torreón. Carretera Torreón-San Pedro km 7.5, Ejido \\ Ana, Torreón, Coahuila, México. CP. 27170. (citlaly_rrha@hotmail.com; fortismanuel@hotmail.com; \\ joorvi66@hotmail.com). ${ }^{2}$ Departamento de Materiales Avanzados-Centro de Investigación en Química \\ Aplicada. Blvd. Enrique Reyna Hermosillo 140, Saltillo, Coahuila, México. CP. 25294. \\ (hortensia.ortega@ciqa.edu.mx). ${ }^{3}$ Universidad Autónoma Agraria Antonio Narro-Departamento de \\ Horticultura. Periférico y Carretera a Santa Fe s/n, Torreón, Coahuila, México. CP. 27000. (juan. \\ manuelnava@hotmail.com). \\ ${ }^{\S}$ Autor para correspondencia: ppreciador@yahoo.com.mx.
}

\section{Resumen}

El uso de nanopartículas de quitosán (NPs CS) se ha vuelto una alternativa prometedora en la agricultura moderna como un inductor en la biosíntesis de metabolitos secundarios. El objetivo del presente trabajo fue evaluar el efecto de NPs CS en la calidad nutraceútica de germinados de triticale (x Triticosecale Wittmack). Dosis creciente de NPs CS: 0, 0.1, 0.2, 0.4 y $0.8 \mathrm{mg} \mathrm{ml}^{-1}$ se aplicaron una sola vez en la etapa de imbibición, después se dejaron en cajas Petri durante 7 días a $25 \pm 2{ }^{\circ} \mathrm{C}$ de temperatura. Las NPs CS no afectaron la germinación ni el peso freso de la raíz a las concentraciones probadas, y a la concentración de $0.1 \mathrm{mg} \mathrm{ml}^{-1}$ aumentó el peso fresco del brote hasta $83.3 \%$. En presencia de $0.8 \mathrm{mg} \mathrm{ml}^{-1}$ de las NPs CS los compuestos fenólicos disminuyen un $7 \%$ y aumentan $29 \%$ los flavonoides. Los resultados confirman un efecto promotor de las NPS CS en los germinados, abriendo la posibilidad de ser utilizadas como inductores en la biosíntesis de compuestos bioactivos en germinados de triticale.

Palabras clave: x Triticosecale Wittmack, metabolitos secundarios, nanotecnología.

Recibido: marzo de 2021

Aceptado: mayo de 2021 


\section{Introducción}

La nanotecnología es una alternativa en la agricultura moderna al producir agroproductos como nanofertilizantes, nanopesticidas, nanoherbicidas y nanosensores, que permiten incrementar el rendimiento de alimentos de manera sustentable y reducir el impacto ambiental (Lira et al., 2018). El uso de nanomateriales es de gran interés para su estudio debido a su tamaño y a las aplicaciones que pueden tener gracias a las propiedades físicas y químicas, que adquieren a escala nanométrica en comparación con el material de tamaño micro (Hojjat y Hojjat, 2015).

Entre estos nanomateriales, las nanopartículas de quitosán (NPs CS), son de gran interés ya que se obtienen gracias a la versatilidad del quitosán y disponibilidad de grupos funcionales (amino, $\mathrm{NH}_{2}$ ) (Salachna y Zawadzińska, 2014; Kumaraswamy et al., 2018), no toxicidad, biocompatibilidad y biodegradabilidad (Divya et al., 2019).

En este sentido las NPs CS se han vuelto una alternativa prometedora en el cebado de semillas, debido a su actividad biológica alta ya que las NPs CS interactúan en conjunto con la célula viva (Pedroso, 2017; Divya y Jisha, 2018; Souza et al., 2019), provocando así la síntesis de varias biomoléculas como inductores que obligan al germinado a reaccionar ante ellos teniendo como consecuencia que desarrolle una mayor síntesis de metabolitos secundarios (Hidangmayum et al., 2019; Paramo et al., 2020), lo cual se ha demostrado en germinados de tomate, arroz y trigo (Colman et al., 2019; Divya et al., 2019; Li et al., 2019).

Por otro lado, los germinados son una fuente de carbohidratos, fibra, vitaminas, nutrimentos esenciales y compuestos bioactivos, los cuales se han relacionado con la prevención y tratamiento de enfermedades. La presencia de estos compuestos, en los germinados, pueden incrementarse por las condiciones de producción, calidad de las semillas y las condiciones de germinación (Dziki et al., 2015).

Recientes avances del uso de la nanotecnología en la agricultura permiten tratar de comprender el papel de las NPs Cs en germinados de triticale (x Triticosecale Wittmack), ya que este presenta actualmente un incremento interesante de $30 \%$ en la producción de alimentos a nivel mundial (Aquino y Gómez, 2019). Las NPs CS son un material prometedor para los tratamientos de semillas, por lo cual el objetivo de este trabajo fue evaluar el efecto de las NPs CS sobre la síntesis de compuestos bioactivos en germinados de triticale.

\section{Materiales y métodos}

Este estudio se realizó en un laboratorio de Biotecnología ubicado en el Instituto Tecnológico de Torreón, México en latitud $24^{\circ} 30^{\prime}$ y 27 latitud norte, $102^{\circ} 00^{\prime}$ y $104^{\circ} 40^{\prime}$ longitud oeste.

\section{Síntesis de nanopartículas de quitosán (NPs CS)}

Las NPs CS fueron sintetizadas por el método de gelación iónica en el Centro de Investigación de Química Aplicada (Saltillo, Coahuila), usando quitosán (Marine, Hydrocolloids, Kerala, India) y tripolifosfato de sodio (TPP) (Sigma-Aldrich, USA) como entrecruzaste en una relación de 10:3 v/v de CS: TPP (Kumaraswamy et al., 2018) con forma esférica y un tamaño de partícula de 111 
$\pm 21 \mathrm{~nm}$, las cuales se caracterizaron mediante UV-vis observándose una absorción de $195 \mathrm{~nm}$ (Figura 1) y por espectrofotometría infrarroja (FTIR por ATR) donde se observaron las bandas características de los grupo amino (NH2) a $3346 \mathrm{~cm}^{-1}$ y carboxilo $(\mathrm{C}=\mathrm{O})$ a $1635 \mathrm{~cm}^{-1}$ (Figura 2), coincidentes a la estructura química del compuesto (Manikandan y Sathiyabama, 2016).

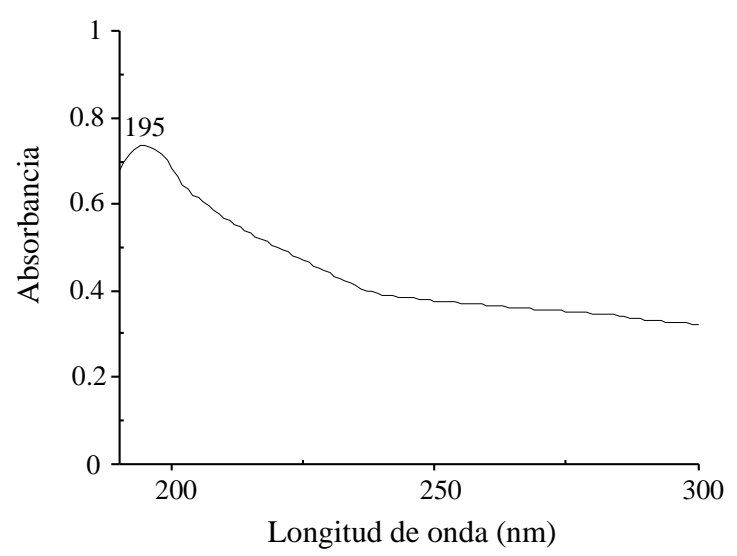

Figura 1. Espectros de absorbancia de NPs CS relación CS:TPP 10:3 v/v.

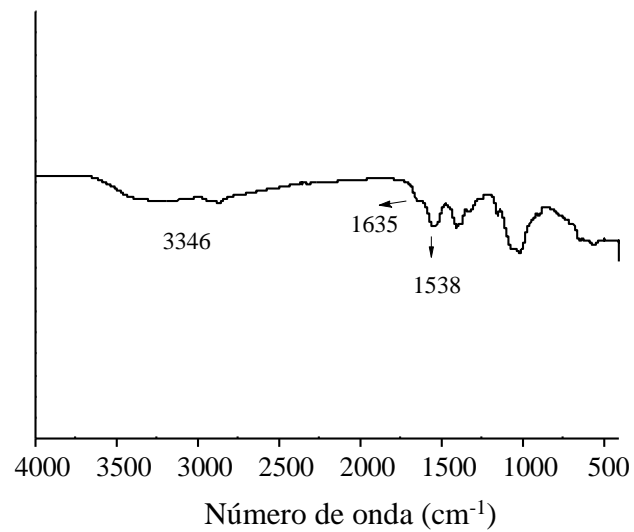

Figura 2. Espectros de FTIR por ATR de NPs CS relación CS:TPP 10:3 v/v.

\section{Material vegetal}

Se utilizaron semillas de triticale (x Triticosecale Wittmack), de tamaño uniforme, que fueron desinfectadas con etanol al $75 \%$ por 5 min y se lavaron 4 veces con agua destilada (Li et al., 2019).

\section{Ensayo de germinación y medición del crecimiento}

Las semillas fueron divididas en cinco tratamientos principales de diez semillas: (testigo) agua destilada, 0.1, 0.2, 0.4 y $0.8 \mathrm{mg} \mathrm{ml}^{-1}$ de NPs CS (Colman et al., 2019; Li et al., 2019), los tratamientos se aplicaron una sola vez a la semilla durante la etapa de imbibición, las semillas de triticale fueron embebidas con las correspondientes soluciones de tratamiento durante $8 \mathrm{~h}$ en la oscuridad. 
Posteriormente para la germinación se colocaron diez semillas por caja Petri, las cuales tenían una doble capa de papel de filtro Whatman \#1 empapado previamente con $5 \mathrm{ml}$ de agua destilada, las cajas Petri se sellaron con cinta adhesiva y se colocaron en una incubadora de crecimiento artificial (HGZ-150) con un ciclo día/noche de $12 \mathrm{~h}$, a $25 \pm 2{ }^{\circ} \mathrm{C}$ respectivamente, con $60 \%$ de humedad relativa (Li et al., 2019).

La germinación de las semillas se calculó diariamente de acuerdo con los lineamientos de la Asociación Internacional de Pruebas de Semillas (ISTA) y se registraron los parámetros de crecimiento durante 7 días. La germinación de la semilla se verificó cuando la longitud del germen alcanzó la mitad de la longitud de la semilla (Faraji et al., 2018).

\section{Parámetros evaluados durante el desarrollo del bioensayo}

\section{Porcentaje de germinación}

Se determinó a los siete días después de la siembra en el segundo conteo, para lo cual se consideró el conteo total de semillas germinadas y el resultado se expresó como se muestra en la siguiente ecuación: porcentaje de germinación $=\left(\frac{\text { número de semillas germinadas }}{\text { número de semillas incubadas }}\right) \times 100$

\section{Vigor de semillas}

Al cuarto día después de la siembra, se realizó el primer conteo para la toma de datos de semillas germinadas (plántulas que tienen bien desarrolladas la raíz y la plúmula, con desarrollo total de 2 $\mathrm{cm}$ en promedio). Para determinar el vigor de la semilla, expresando el resultado en porcentaje

según la fórmula: vigor de semilla $=\left(\frac{\text { plántulas normales }}{\text { número de semillas incubadas }}\right) \times 100$

\section{Peso fresco, brote y raíz}

Se registró el peso freso del brote y la raíz en una balanza analítica (ADN modelo HR-200 ${ }^{\circledR}$ ) para determinar el valor de la biomasa fresca y se reportó en miligramos por germinado (Martinez et al., 2019).

\section{Pigmentos fotosintéticos}

El contenido de clorofila $(\mathrm{Chl})$ en germinados de triticale se realizó de acuerdo con el método descrito por Liu et al (Liu et al., 2013). Para lo cual se pesaron $0.5 \mathrm{~g}$ de germinados, que fueron homogenizados en un mortero con $10 \mathrm{ml}$ de etanol al 95\%. El homogeneizado se centrifugó a 1 $500 \mathrm{rpm}$ durante $20 \mathrm{~min}$ y se recogió el sobrenadante, para luego medir la absorbancia a absorbancia a 665 y $649 \mathrm{~nm}$, respectivamente. El contenido de Chl se calculó de acuerdo con la fórmula $\quad$ siguiente: $\quad \mathrm{Chl}(\mathrm{a})=1395 \mathrm{~A}_{665}-6.88 \mathrm{~A}_{649} . \mathrm{Chl}(\mathrm{b})=24.95 \mathrm{~A}_{649}-7.32 \mathrm{~A}_{665}$. $\operatorname{Chl}(a+b)=\operatorname{Chl}(a)+\operatorname{Chl}(b)$.

Contenido de Chl $\left(\frac{\mathrm{mg}}{\mathrm{g}} \mathrm{PF}\right)=\frac{\operatorname{Chl}(\mathrm{a}+\mathrm{b}) * \text { Volumen de la extraccion }(\mathrm{mL}) * \text { tiempo de la dilucion }}{\text { Peso fresco }(\mathrm{g})}$ 


\section{Preparación de extractos para la calidad nutracéutica}

En la obtención de extractos se mezclaron $2 \mathrm{~g}$ de muestra fresca en $10 \mathrm{ml}$ de etanol al 80\%, con agitación orbital constante por $24 \mathrm{~h}$ a $70 \mathrm{rpm}$ y $5{ }^{\circ} \mathrm{C}$. Después los extractos fueron centrifugados a 3000 rpm durante 5 min y el sobrenadante fue extraído para su análisis (Salas et al., 2016).

\section{Contenido total de fenoles}

Se determinó mediante una modificación del método Folin-Ciocaltea (Singleton et al., 1999), se tomaron $50 \mu \mathrm{L}$ del extracto etanoico, diluidos en $3 \mathrm{ml}$ de agua destilada y se añadieron $250 \mu \mathrm{l}$ del reactivo Folin-Ciocalteau (1N), se agitó y dejó reaccionar durante $3 \mathrm{~min}$. Posteriormente $750 \mu \mathrm{l}$ de $\mathrm{Na}_{2} \mathrm{CO}_{3}(20 \%)$ y $950 \mu \mathrm{l}$ de agua destilada fueron añadidos, la solución se dejó reposar durante $2 \mathrm{~h}$, para luego ser cuantificadas en un espectrofotómetro UV -Vis a $760 \mathrm{~nm}$. Se usaron soluciones de ácido gálico para construir la curva de calibración. Los resultados se expresaron como mg equivalentes de ácido gálico (AGE)/100 g de peso fresco.

\section{Flavonoides totales}

Fueron determinados por método colorimétrico (Colina, 2016), se tomaron $250 \mu 1$ de extracto etanólico, se mezclaron con $1.25 \mathrm{ml}$ de agua destilada y $75 \mu 1$ de NaNO2 (5\%). Después de 5 min de reposo, se agregaron $150 \mu \mathrm{l}$ de $\mathrm{AlCl} 3$ (cloruro de aluminio-1-etil-3-metilimidazolio cloruro (Sigma-Aldrich, St. Louis, MO, EE. UU.).

Posteriormente $500 \mu \mathrm{L}$ de $\mathrm{NaOH}(1 \mathrm{M})$ y $275 \mu \mathrm{l}$ de agua destilada se añadieron, las muestras se agitaron vigorosamente, para luego ser cuantificadas en un espectrofotómetro UV-Vis a $510 \mathrm{~nm}$. El estándar se preparó con quercetina disuelta en etanol absoluto para obtener la curva de calibración. Los resultados se expresaron en mg QE/100 g peso fresco.

\section{Análisis estadístico}

El experimento se realizó mediante un diseño completamente al azar con cinco tratamientos y diez repeticiones. Los resultados obtenidos se analizaron mediante análisis de varianza y comparación de medias con la prueba de Tukey $(p \leq 0.05)$ utilizando el paquete estadístico Statistical Analysis System Institute (SAS) versión 9.3. La normalidad de los datos para cada variable de respuesta se verificó con el test de Kolmogorov-Smirnov, los datos de porcentaje de germinación y las variables de capacidad antioxidante (ambas expresadas como porcentaje) se normalizaron aplicando la transformación de arcoseno y raíz cuadrada.

\section{Resultados y discusión}

\section{Germinación de las semillas}

El porcentaje de germinación y el peso fresco son unas de las principales propiedades implicadas en la calidad fisiológica de la semilla (Morales et al., 2017). Los resultados del presente trabajo muestran que las variables del porcentaje de germinación y el peso fresco de la raíz no mostraron diferencia significativa $(p>0.05)$ a las diferentes concentraciones que fueron aplicadas de NPs CS; sin embargo, causaron diferencia significativa en el vigor y el peso fresco del brote (Cuadro 1), 
siendo que el vigor de la semilla mostró una disminución de $9.75 \%\left(0.1 \mathrm{mg} \mathrm{ml}^{-1}\right)$, contrario a lo que reportaron Colman et al. (2019) en semillas germinadas de tomate tratadas con $0.1 \mathrm{mg} \mathrm{ml}^{-1}$, con efectos positivos en el índice de vigor respecto al control.

Cuadro 1. Comparación de medias para el porcentaje de germinación y vigor, así peso fresco del brote y de raíz de semillas de triticale tratadas con NP CS.

\begin{tabular}{|c|c|c|c|c|}
\hline \multirow{2}{*}{$\begin{array}{l}\text { NPs CS } \\
\left(\mathrm{mg} \mathrm{ml}^{-1}\right)\end{array}$} & Germinación & Vigor & Peso fresco de brote & Peso fresco de raíz \\
\hline & \multicolumn{2}{|c|}{$(\%)$} & \multicolumn{2}{|c|}{$(\mathrm{mg})$} \\
\hline Control & $86 \pm 0.89 a$ & $82 \pm 0.81 \mathrm{a}$ & $38.7 \pm 0.32 b$ & $74.2 \pm 0.28 \mathrm{a}$ \\
\hline 0.1 & $82 \pm 0.85 \mathrm{a}$ & $74 \pm 0.86 \mathrm{c}$ & $70.4 \pm 0.15 \mathrm{a}$ & $79.6 \pm 0.98 \mathrm{a}$ \\
\hline 0.2 & $96 \pm 1.02 \mathrm{a}$ & $80 \pm 0.8 \mathrm{ab}$ & $55.2 \pm 0.25 \mathrm{ab}$ & $53.2 \pm 0.31 \mathrm{a}$ \\
\hline 0.4 & $88 \pm 1.05 \mathrm{a}$ & $76 \pm 0.84 \mathrm{c}$ & $64.8 \pm 0.58 \mathrm{a}$ & $73.8 \pm 0.42 \mathrm{a}$ \\
\hline 0.8 & $88 \pm 0.98 \mathrm{a}$ & $80 \pm 0.82 \mathrm{ab}$ & $56.0 \pm 0.35 b$ & $56.8 \pm 0.45 \mathrm{a}$ \\
\hline
\end{tabular}

Valores con letras iguales en cada columna, son iguales de acuerdo con la prueba de Tukey $(p \leq 0.05)$. Los valores son el promedio de cinco repeticiones. Medias $(\mathrm{n}=5) \pm$ desviación estándar.

En cuanto a la acumulación de biomasa el peso fresco del brote aumento hasta $81 \%$ con la dosis de $0.1 \mathrm{mg} \mathrm{ml}^{-1}$ lo que confirma el efecto positivo de las NPs CS a concentraciones bajas sobre la germinación de las semillas de triticale, dicho efecto podría atribuirse a la capacidad estimulante de la actividad metabólica de las NPs CS, logrando un aumento del potencial intrínseco en el desarrollo de la semilla con la absorción de las NPs CS (Divya et al., 2019; López et al., 2019), ya sea por imbibición, recubrimiento o cebado de las semillas (Costales et al., 2020).

Diversos estudios con concentraciones altas de NPs Cs o CS han descrito una inhibición del crecimiento de las raíces y alternativamente una promoción en la aparición de un número mayor de raíces seminales (Colman et al., 2019; López et al., 2019), como se muestra en la Figura 3 atribuyéndoselo a la estimulación de la síntesis de fitohormonas (López et al., 2019) y a la activación de genes de defensa (Rodríguez et al., 2019) como se ha observado en cultivos como tomate (Colman et al., 2019; Solórzano, 2019), salicornia bigelovii (López et al., 2020) (Lanchimba, 2019), sorgo (Holguin et al., 2020), arroz (Divya et al., 2019) y trigo (Li et al., 2019).

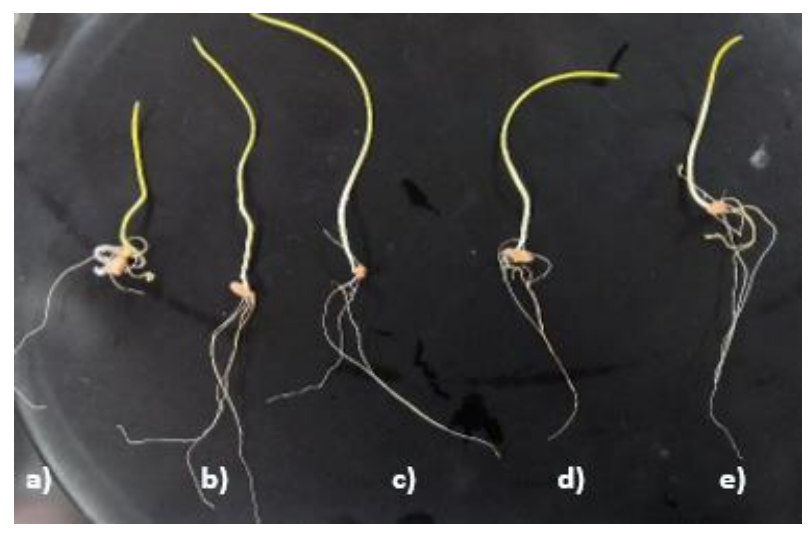

Figura 3. Germinación de semillas de triticale en respuesta a la aplicación de NPs CS en concentraciones de: a) control; b) 0.1 ; c) 0.2 ; d) 0.4 ; y e) $0.8 \mathrm{mg} \mathrm{ml}^{-1}$. 


\section{Pigmentos fotosintéticos}

El contenido de Chl de los germinados mostró que la dosis de $0.2 \mathrm{mg} \mathrm{ml}^{-1}$ de NPs CS tienen efecto significativo, ya que incrementó 59\% sobre el control (Figura 4). Según el trabajo reportado por Acharya et al. (2020), mencionan que el uso de nanopartículas en cebado de semillas causara toxicidad en los germinados a altas concentraciones, pudiéndose observar una disminución en los pigmentos fotosintéticos (Acharya et al., 2020) por la ruptura de la clorofila y la inhibición de la enzima p-hidroxifenilpiruvato en la biosíntesis de clorofila (Miras, 2018), causado por el estrés provocado por las nano partículas.

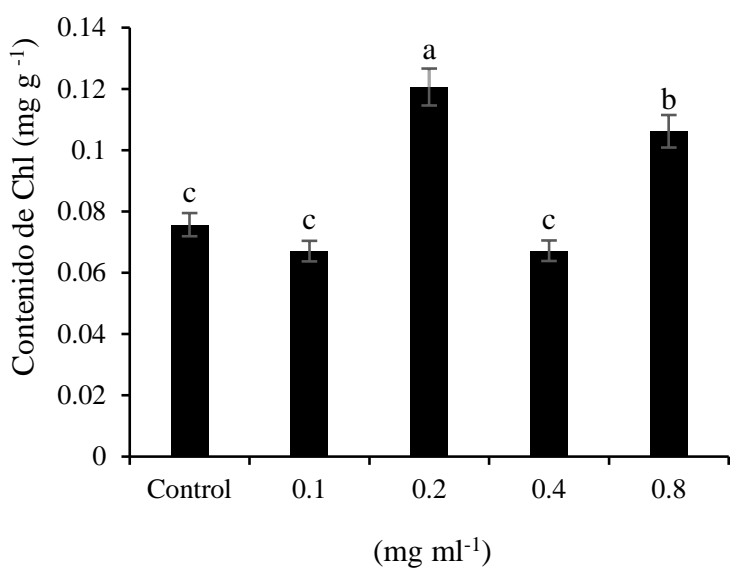

Figura 4. Efecto de las NPs CS en el contenido de clorofila en germinados de trigo. Los valores promedio en las columnas con diferentes literales difieren estadísticamente entre ellos (Tukey $p \leq$ $0.05)$.

Por lo contrario, en este trabajo se demostró que el cebado con las NPs CS, aumenta los pigmentos fotosintéticos en bajas concentraciones en comparación con el control. Sin embargo, es difícil establecer un modelo de respuesta de los efectos de las NPs en los germinados ya que el efecto depende de la especie, la concentración y el tipo de NPs (Arruda et al., 2015).

\section{Calidad nutraceútica: fenoles y flavonoides totales}

La utilización de NPs CS puede actuar como agente inductor de procesos metabólicos ya que pueden incrementar el contenido de compuestos bioactivos destacado en el desarrollo y efecto estimulante de la producción de metabolitos secundarios (Xoca et al., 2017; Xoca et al., 2019; Montalvo et al., 2020).

Los resultados de la presente investigación demuestran que las variables relacionadas con la calidad nutraceútica en el germinado de triticale: fenoles y flavonoides totales son afectados por la dosis alta aplicada de las NPs CS $\left(0.8 \mathrm{mg} \mathrm{ml}^{-1}\right)$ los compuestos fenólicos disminuyeron respecto al control hasta 7\% (Figura 5a), y los flavonoides aumentaron en 29\% respecto al control (Figura 5b), lo que corrobora el efecto positivo de las NPs CS sobre los germinados de triticale. 
a)

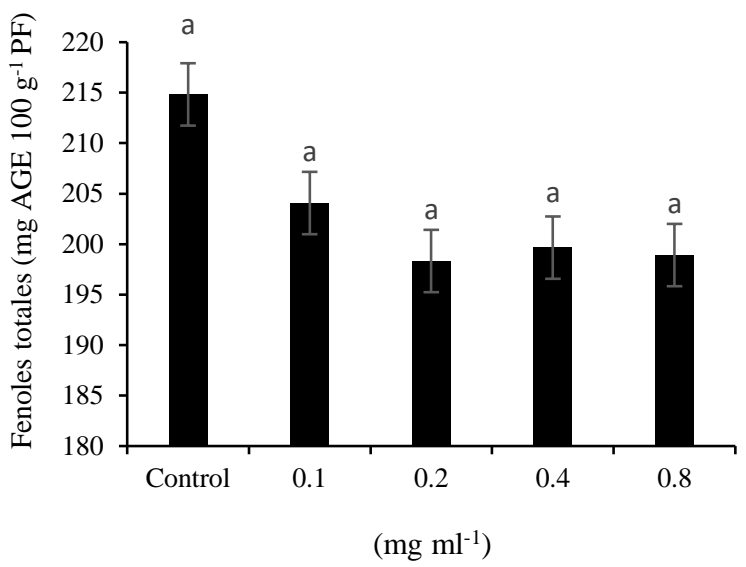

b)

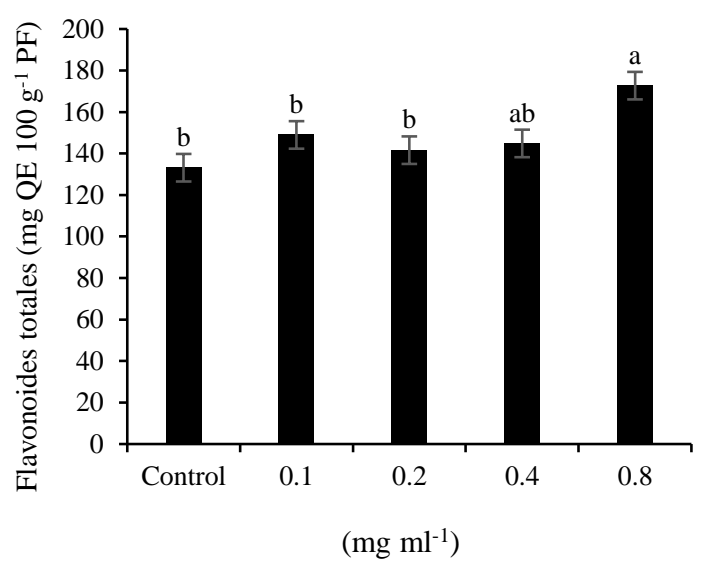

Figura 5. Efecto de NPs CS sobre el contenido de compuestos fenólicos totales (a), flavonoides totales (b) en germinados de triticale.

Dichos resultados pueden atribuirse a que las altas concentraciones de NPs CS inducen a una explosión oxidativa inicial con acumulación de peróxido de hidrógeno $\left(\mathrm{H}_{2} \mathrm{O}_{2}\right)$ (Martínez et al., 2015), y se cree que esto puede conducir a la inducción de enzimas de defensa de las plantas y a la síntesis de metabolitos secundarios, como los polifenoles, lignina, flavonoides y fitoalexinas (Malerba y Cerana, 2016) mejorando las respuestas de defensa ante el estrés biótico y abiótico (Hidangmayum et al., 2019).

Pudiendo ser una alternativa viable para mejorar las propiedades funcionales y biológicas del germinado (Rodríguez et al., 2019); sin embargo, es difícil establecer los efectos de las NPs CS en los germinados ya que varían según la especie vegetal, las etapas de crecimiento, la dosis y a la exposición de las NPs (Medina, 2017).

\section{Conclusiones}

La aplicación de las NPs CS en germinados de triticale a dosis de $0.1 \mathrm{mg} \mathrm{ml}^{-1}$ afecto el peso fresco de brote, pero no el peso fresco de raíz, mientras que dosis de $0.2 \mathrm{mg} \mathrm{ml}^{-1}$ mostró los mejores resultados en porcentaje de vigor y contenido de pigmentos fotosintéticos, lo cual confirma que el cebado de semillas con NPs CS no provoco efectos negativos, además dosis altas de $0.8 \mathrm{mg} \mathrm{ml}^{-1}$ de NPs CS afectan la calidad nutraceútica aumentando el contenido de flavonoides en germinados de triticale.

Las NPs CS podrían ser una buena alternativa para mejorar la calidad de los germinados; sin embargo, se necesita más investigación para aclarar los efectos de los NPs CS ya que existen factores que dependen de la especie y la concentración. 


\section{Literatura citada}

Acharya, P.; Jayaprakasha, G. K.; Crosby, K.; Jifon, J. L. and Patil, B. S. 2020. Nanoparticlemediated seed priming improves germination, growth, yield, and quality of watermelons (Citrullus lanatus) at multi-locations in Texas. Scientific Reports. 10(1):1-16.

Aquino, V. C. y Gómez, N. I. 2019. Triticale (x Triticosecale Wittmack): bioestimulantes orgánicos y fertilización nitrogenada sobre los componentes de rendimiento forrajero en campaña chica-valle del mantaro. Scientia Agropecuaria. 10(4):469-477.

Arruda, S. C.; Diniz, A. L.; Moreto, R.; Antunes, R. and Zezzi, A. 2015. Nanoparticles applied to plant science: a review. Talanta. 131(1):693-705.

Colina, A. C. 2016. Análisis fitoquímico, determinación cualitativa y cuantitativa de flavonoides y taninos, actividad antioxidante, antimicrobiana de las hojas de "Muehlenbeckia hastulata (JE Sm) IM Johnst" de la zona de Yucay (Cusco). Tesis Profesional de Químico. Universidad Nacional Mayor de San Marcos. Lima, Perú.

Colman, S. L.; Salcedo, M. F.; Mansilla, A. Y.; Iglesias, M. J.; Fiol, D. F.; Saldaña, S. M.; Alvarez, V. A.; Chevalier, A. A. and Casalongué, C. A. 2019. Chitosan microparticles improve tomato seedling biomass and modulate hormonal, redox and defense pathways. Plant Physiol. Biochem. 143(1):203-211.

Costales, D.; Falcón, A. B. y Travieso, L. 2020. Efecto de la masa molecular de quitosanos en la germinación y el crecimiento in vitro de soya. Cultivos Tropicales. 41(1):1-10.

Divya, K. and Jisha, M. 2018. Chitosan nanoparticles preparation and applications. Environ. Chem. Letters 16(1):101-112.

Divya, K.; Vijayan, S.; Nair, S. J. and Jisha, M. 2019. Optimization of chitosan nanoparticle synthesis and its potential application as germination elicitor of Oryza sativa L. Inter. J. Biol. Macromol. 124(1):1053-1059.

Dziki, D.; Gawlik, U.; Kordowska, M. and Domań, M. 2015. Influence of elicitation and germination conditions on biological activity of wheat sprouts. J. Chem. 7:1-8.

Faraji, J.; Sepehri, A. and Salcedo, J. C. 2018. Titanium dioxide nanoparticles and sodium nitroprusside alleviate the adverse effects of cadmium stress on germination and seedling growth of wheat (Triticum aestivum L.). Universitas Scientiarum. 23(1):61-87.

Hidangmayum, A.; Dwivedi, P.; Katiyar, D. and Hemantaranjan, A. 2019. Application of chitosan on plant responses with special reference to abiotic stress. Physiol. Mol. Biol. Plants. 25(2):313-326.

Hojjat, S. S. and Hojjat, H. 2015. Effect of nano silver on seed germination and seedling growth in fenugreek seed. Inter. J. Food Eng. 1(2):106-110.

Holguin, R. J.; Vargas, J. M.; López, G. A.; Rodríguez, F.; Borbón, C. G. y Rueda, E. O. 2020. Efecto de quitosano y consorcio simbiótico benéfico en el rendimiento de sorgo en la zona indígena "Mayos" en Sonora. Terra Latinoam. 38(3):705-714.

Kumaraswamy, R.; Kumari, S.; Choudhary, R. C.; Pal, A.; Raliya, R.; Biswas, P. and Saharan, V. 2018. Engineered chitosan based nanomaterials: Bioactivities, mechanisms and perspectives in plant protection and growth. Int. J. Food Eng. 113(1):494-506.

Lanchimba, W. I. 2019. Evaluación de quitosano en el crecimiento y desarrollo de tomate (Solanum lycopersicum, L.) en condiciones de casa de cultivo. Cuba: bayamo: Universidad Técnica de Cotopaxi (UTC). Tesis de Ingeniero Agrónomo. Universidad Técnica de Cotopaxi. Bayamo, Cuba. 
Li, R.; He, J.; Xie, H.; Wang, W.; Bose, S. K.; Sun, Y.; Hu, J. and Yin, H. 2019. Effects of chitosan nanoparticles on seed germination and seedling growth of wheat (Triticum aestivum L.). Int. J. Biol. Macromol. 126(1):91-100.

Lira, R. H.; Méndez, B.; De-Santos, G. y Vera, R. I. 2018. Potencial de la nanotecnología en la agricultura. Acta Universitaria. 28(2):9-24.

Liu, H.; Zhang, Y. H.; Yin, H.; Wang, W. X.; Zhao, X. M. and Du, Y. G. 2013. Alginate oligosaccharides enhanced Triticum aestivum L. tolerance to drought stress. Plant Physiology and Biochemistry. 62(1):33-40.

López, B.; Mondaca, I.; Gortáres, P.; Meza, M.; Balderas, J. d. J.; Ruíz, C. and Rueda, E. O. J. 2020. Ecofisiología y bioquímica de salicornia bigelovii (Torr.) por efecto de quitosanoaib bajo condiciones del desierto de sonora. Polibotánica. 49(5):75-92.

López, B. E.; Mondaca, I.; Gortáres, P.; Meza, M. M.; Balderas, J. d. J.; Ruiz, C. y Rueda, E. O. J. 2019. Enraizamiento de esquejes de Salicornia bigelovii (Torr.) por quitosano como un bioproducto de origen marino. Terra Latinoam. 37(4):361-369.

Malerba, M. and Cerana, R. 2016. Chitosan effects on plant systems. Int. J. Mol. Sci. 17(7):9961011.

Manikandan, A. and Sathiyabama, M. 2016. Preparation of chitosan nanoparticles and its effect on detached rice leaves infected with Pyricularia Grisea. Inter. J. Biol. Macromolecules. 84(1):58-61.

Martinez, B. M. E.; Brown, A. y Coria, C. 2019. Contenido de hierro, calcio y magnesio durante el proceso de producción de germinados de lentejas (lens culinaris) bajo cultivo aeropónico. Universidad Nacional de Cuyo. Facultad de Ciencias Agrarias. Tesis de Licenciatura en Bromatología. Universidad Nacional de Cuyo. Mendoza, Argentina.

Martínez, L.; Reyes, Y.; Falcón, A. y Núñez, M. 2015. Efecto del tratamiento a las semillas con quitosana en el crecimiento de plántulas de arroz (Oryza sativa L.) cultivar INCA LP-5 en medio salino. Cultivos Tropicales. 36(1):143-150.

Medina, E. 2017. Diseño y evaluación de recubrimientos en base a proteínas de quínoa y quitosano que contienen agentes naturales nanoparticulados para su aplicación en frutillas. Tesis de Doctorado en Nutrición y Alimentos. Facultad de Ciencias Químicas y Farmacéuticas. Universidad de Chile. Santiago, Chile.

Miras, M. B. 2018. Estudio metabolómico y genómico de cultivos celulares de zanahoria. Tesis de Doctorado. Facultad de Biología Universidad de Murcia. Murcia, España.

Montalvo, E.; Romero, R.; Sánchez, J. A.; Ruvalcaba, J. M.; Pérez, A. y Anaya, L. M. 2020. Functionalization of edible coating chitosan-based for fruits and vegetables postharvest preservation. TIP Rev. Especializada en Ciencias Químico-Biológicas. 23(1):1-14.

Morales, M. E.; Peña, C. B.; García, A.; Aguilar, G. y Kohashi, J. 2017. Características físicas y de germinación en semillas y plántulas de frijol (Phaseolus vulgaris L.) silvestre, domesticado y su progenie. Agrociencia. 51(1):43-62.

Paramo, L. A.; Feregrino, A. A.; Guevara, R.; Mendoza, S. and Esquivel, K. 2020. Nanoparticles in agroindustry: applications, toxicity, challenges, and trends. Nanomaterials. 10(9):1654-1687.

Pedroso, S. 2017. Modificaciones físico-químicas de nanopartículas de diamante y oro en la formación de nanoestructuras híbridas para uso en la biomedicina. Tesis de Doctorado. Universidad de Sonora. Sonora, México.

Rodríguez, C. A.; González, R. R.; Bautista, S. y Gutiérrez, P. 2019. Efecto del quitosano en el control de Alternaria sp. en plantas de jitomate en invernadero. TIP. Revista especializada en ciencias químico-biológicas. 22(1):1-7. 
Salachna, P. and Zawadzińska, A. 2014. Effect of chitosan on plant growth, flowering and corms yield of potted freesia. J. Ecol. Eng. 15(3):97-102.

Salas, L.; Gaucín, J. M.; Preciado, P. M.; Fortis, M.; Valenzuela, J. R y Ayala, A. V. 2016. Efecto del ácido benzoico en la capacidad antioxidante de germinados de trigo. Rev. Mex. Cienc. Agríc. 17(3):3397-3404.

Singleton, V. L.; Orthofer, R. and Lamuela, R. M. 1999. Analysis of total phenols and other oxidation substrates and antioxidants by means of folin-ciocalteu reagent. Methods Enzymol. 299(7):152-178.

Solórzano, A. E. 2019. Efecto de quitosano, hongos micorrízicos y ácidos húmicos sobre el crecimiento y desarrollo en variedades de pimiento (Capcicum annuum $\mathrm{L}$ ) bajo condiciones protegidas. Tesis de Ingeniero Agrónomo. Departamento y Universidad Quevedo-UTEQ. Quevedo, Ecuador.

Souza, J. P.; Cayaso, R.; Martín, P.; Pereira, R.; Fernandes, J. and Martín, J. 2019. Efectos inmediatos y tras almacenaje del recubrimiento con boro de semillas de algodón. In: X Congreso Ibérico de Agroingeniería. 1199-1208 pp.

Xoca, L. Á.; Cuellar, E. A.; González, S.; Gutiérrez, P.; López, U.; Herrera, L.; Vega, J. and Chacón, A. 2017. Transcriptomic analysis of avocado hass (Persea americana Mill) in the interaction system fruit-chitosan-Colletotrichum. Frontiers Plant Sci. 8(1):956-969.

Xoca, L. Á.; Aguilera, S.; Vega, J.; Acevedo, G.; Tovar, E.; Stoll, A.; Herrera, L. and Chacón, A. 2019. Activation of the phenylpropanoid biosynthesis pathway reveals a novel action mechanism of the elicitor effect of chitosan on avocado fruit epicarp. Food Res. Inter. 121(1):586-592. 